\title{
Arterial stiffness and atrial fibrillation recurrence: another risk marker or a call for better management of concomitant disease?
}

\author{
J. R. de Groot · D. Linz
}

Accepted: 3 March 2022 / Published online: 8 March 2022

(C) The Author(s) 2022

Atrial fibrillation (AF) is the most common cardiac arrhythmia, and it is associated with increased risk of ischaemic stroke, heart failure and mortality [1]. Cardiovascular risk factors are highly prevalent in patients with AF and vice versa [2]. In the European Society of Cardiology Guidelines for the diagnosis and management of AF, systematic assessment and management of modifiable concomitant cardiovascular risk factors are important pillars of the ABC approach (Anticoagulation/Avoid stroke-Better symptom control-Comorbidities/Cardiovascular risk factor management) [1]. In addition to oral anticoagulation for prevention of stroke, better management of symptoms by heart rate control and restoration of sinus rhythm with antiarrhythmic drugs or through catheter ablation is recommended. Despite enormous progress in ablation technology, interventional treatment of AF with catheter ablation remains a challenge, in particular when the absence of AF during follow-up is considered as an endpoint [3].

\footnotetext{
J. R. de Groot (凶)

Department of Cardiology, Heart Centre, Amsterdam University Medical Centres, University of Amsterdam, Amsterdam, The Netherlands

j.r.degroot@amsterdamumc.nl

D. Linz

Department of Cardiology, Cardiovascular Research Institute Maastricht, Faculty of Health, Medicine and Life Sciences, Maastricht University and Maastricht University Medical Centre+, Maastricht, The Netherlands

Department of Cardiology, Radboud University Medical Centre, Nijmegen, The Netherlands

Department of Biomedical Sciences, University of Copenhagen, Copenhagen, Denmark

Centre for Heart Rhythm Disorders, University of Adelaide and Royal Adelaide Hospital, Adelaide, Australia
}

Several factors have been identified as predictors of AF recurrence after AF ablation. For example, the APPLE score (attributing one point each to age $>65$ years, persistent AF, impaired estimated glomerular filtration rate $<60 \mathrm{ml} /$ minute per $1.73 \mathrm{~m}^{2}$, left atrial diameter $\geq 43 \mathrm{~mm}$, left ventricular ejection fraction (LVEF) $<50 \%$ ) has been introduced as a tool to estimate the risk of $\mathrm{AF}$ recurrence after $\mathrm{AF}$ ablation. However, aside from the statistically significant predictive value, the accuracy of this score is modest at best [4]. Currently established risk factors for AF recurrence that are commonly used in clinical practice to stratify patients for AF ablation include obesity, left atrial volume index (LAVI), $\mathrm{CHA}_{2} \mathrm{DS}_{2}$-VASc score and presence of persistent or long-standing persistent AF. Additionally, surrogate markers of arterial stiffness, such as pulse pressure, pulse wave velocity and reduced aortic compliance, have been described as independent risk factors for AF [5-7]. Despite evolving evidence supporting the association between AF and arterial stiffness, the association between arterial stiffness and AF recurrence after AF ablation remains unclear.

In this issue of the Netherlands Heart Journal, Shchetynska-Marinova and colleagues present their study on the relation of arterial stiffness with AF recurrence after ablation [8]. They report on 151 patients undergoing AF ablation from June 2015 through December 2017 (mean \pm standard deviation age $72 \pm 10$ years, $64 \%$ male, $39 \%$ persistent AF) in whom arterial stiffness was assessed by measuring aortic distensibility. Mean $\mathrm{CHA}_{2} \mathrm{DS}_{2}$-VASc score was $3.0 \pm 1.7$, indicating this cohort of patients were not only relatively old-compared with the Dutch situation-but they were also more severely burdened by cardiovascular comorbidities. As a comparison, a recent analysis of the Netherlands Heart Registration database showed that in the Netherlands, the mean age of pa- 
tients undergoing AF ablation is $60 \pm 10$ years and the mean $\mathrm{CHA}_{2} \mathrm{DS}_{2}$-VASc score is $1.5 \pm 1.3$ [9].

In the study by Shchetynska-Marinova et al., hypertension was present in $78 \%$ of the patients, heart failure in $43 \%$, chronic kidney disease in $32 \%$, coronary artery disease in $29 \%$ and diabetes mellitus in $13 \%$ [8]. The authors calculated aortic distensibility by dividing the difference between systolic and diastolic diameter of the descending aorta (measured by periprocedural transoesophageal echocardiography) by the diastolic diameter and then multiplying that by the pulse pressure (measured by brachial blood pressure) [8]. Aortic distensibility was used as a proxy for arterial stiffness throughout the body. Pulmonary vein isolation was performed with voltage abatement and exit block as procedural endpoints. Patients were followed up for a median duration of 21 months (interquartile range 15-31), with routine visits at the outpatient clinic and 72-hour Holter monitoring once (three months after the procedure).

During follow-up, $62.3 \%$ of the patients experienced AF recurrence [8]. These patients were older, had a higher $\mathrm{CHA}_{2} \mathrm{DS}_{2}$-VASc score, a higher APPLE score, a higher symptom burden (as assessed by European Heart Rhythm Association score), more chronic kidney disease, a lower LVEF and a larger LAVI and more often took digoxin or amiodarone than those without recurrences. Aortic distensibility was significantly lower in patients with AF recurrence than in those without $\left(1.5 \pm 0.7\right.$ vs $2.6 \pm 2.3 \times 10^{-3} \mathrm{~mm} \mathrm{Hg}^{-1}$, $p<0.0001$ ). In multivariable analysis, LAVI (odds ratio (OR) 2.9, 95\% confidence interval (CI) 1.2-3.4) and aortic distensibility (OR 3.6, 95\% CI 2.8-4.1) remained significant predictors of AF recurrence.

How should we appreciate these findings? The authors make a strong case that aortic distensibility-the proxy they used for arterial stiffness-is an independent predictor of AF recurrence after AF ablation in a cohort of patients heavily burdened by cardiovascular comorbidities. This is true from a statistical point of view, but does it also make sense from a biological perspective?

Aortic distensibility strongly correlated with age, hypertension, kidney failure and heart failure but also with LVEF, LAVI and $\mathrm{CHA}_{2} \mathrm{DS}_{2}$-VASc score. In other words: Does aortic distensibility represent a marker of the underlying cardiovascular disease? In that respect, the finding that aortic distensibility correlated with AF outcome after ablation is not very surprising. How aortic distensibility relates to sex is not reported, but a recent subanalysis of the RACE V (Reappraisal of Atrial Fibrillation: Interaction between HyperCoagulability, Electrical remodelling, and Vascular Destabilisation in the Progression of AF) trial showed that biomarkers of vascular remodelling were predominantly increased in male subjects with paroxysmal $\mathrm{AF}$, whereas females expressed more biomarkers of inflammation [10]. Hence, the role of vascular disease in
AF pathophysiology and AF recurrence after ablation may be different for the sexes.

A second consideration is how to appreciate the implication of the predictive value of aortic distensibility for AF recurrence. Is this yet another marker of cardiovascular comorbidity, or does arterial stiffness play a pivotal role in the haemodynamics causing the development and perpetuation of AF, as ShchetynskaMarinova et al. suggest? It remains unclear whether early management of underlying concomitant cardiovascular diseases is effective in preventing the progression of increased aortic distensibility (and decreased arterial stiffness). Another important question is whether and to which extent already existing decreased aortic distensibility (in this study, baseline aortic distensibility was $1.9 \pm 1.1 \times 10^{-3} \mathrm{~mm} \mathrm{Hg}^{-1}$ ) is reversible when potentially modifiable risk factors are managed and controlled.

These questions require further prospective intervention studies and go beyond the current study by Shchetynska-Marinova et al. It remains unclear to which extent the findings in this selected population sample reported by the authors can be extrapolated to a group of consecutive patients referred for AF ablation and how we should appreciate aortic distensibility as a risk marker of AF progression in patients who will not undergo ablation.

In summary, arterial stiffness is associated with the presence of concomitant cardiovascular risk factors and may help to identify AF patients with a higher risk of AF recurrence after ablation. For now, the clear recommendation by current $\mathrm{AF}$ management guidelines that cardiovascular comorbidities need to be optimised as part of a holistic approach towards the AF patient remains unchanged. Future intervention studies are needed to determine whether arterial stiffness is a marker of underlying cardiovascular risk factors, which should trigger a comprehensive risk factor management approach, or whether it represents an independent, modifiable risk factor for recurrence of $\mathrm{AF}$ in patients undergoing AF ablation [1].

Conflict of interest J.R. de Groot has received research grants through his institution from Atricure, Bayer, Boston Scientific, Daiichi Sankyo, Johnson \& Johnson and Medtronic, and honoraria/speaker's fees from AtriCure, Bayer, BIOTRONIK, CardioVasculair Onderwijs Instituut, Daiichi Sankyo, Medtronic, Novartis and Servier. D. Linz has received research grants through his institution from Bayer and Biosense Webster, and honoraria/speaker's fees from Daiichi Sankyo, Medtronic, BIOTRONIK, Respicardia and Itamar Medical.

Open Access This article is licensed under a Creative Commons Attribution 4.0 International License, which permits use, sharing, adaptation, distribution and reproduction in any medium or format, as long as you give appropriate credit to the original author(s) and the source, provide a link to the Creative Commons licence, and indicate if changes were made. The images or other third party material in this article are included in the article's Creative Commons licence, unless indicated otherwise in a credit line to the material. If material is not included in the article's Creative Commons licence and 
your intended use is not permitted by statutory regulation or exceeds the permitted use, you will need to obtain permission directly from the copyright holder. To view a copy of this licence, visit http://creativecommons.org/licenses/by/4.0/.

\section{References}

1. Hindricks G, Potpara T, Dagres N, et al. 2020 ESC Guidelines for the diagnosis and management of atrial fibrillation developed in collaboration with the European Association of Cardio-Thoracic Surgery (EACTS). Eur Heart J. 2021;42:373-498.

2. Fauchier L, Boriani G, de Groot JR, Kreutz R, Rossing P, Camm AJ. Medical therapies for prevention of cardiovascular and renal events in patients with atrial fibrillation and diabetes mellitus. Europace. 2021;23:1873-91.

3. Calkins H, Hindricks G, Cappato R, et al. 2017 HRS/EHRA/ECAS/APHRS/SOLAECE expert consensus statement on catheter and surgical ablation of atrial fibrillation: executive summary. Europace. 2017;20:157-208.

4. Kornej J, Hindricks G, Shoemaker MB, et al. The APPLE score: a novel and simple score for the prediction of rhythm outcomes after catheter ablation of atrial fibrillation. Clin Res Cardiol. 2015;104:871-6.
5. Lau DH, Middeldorp ME, Brooks AG, et al. Aortic stiffness in lone atrial fibrillation: a novel risk factor for arrhythmia recurrence. Plos One. 2013;8:e76776.

6. Chen LY, Leening MJ, Norby FL, et al. Carotid Intimamedia thickness and arterial stiffness and the risk of atrial fibrillation: the atherosclerosis risk in communities (ARIC) study, multi-ethnic study of atherosclerosis (MESA), and the rotterdam study. JAm Heart Assoc. 2016;5:e2907.

7. Kranert M, Shchetynska-Marinova T, Berghoff T, et al. Arterial stiffness is associated with increased symptom burden in patients with atrial fibrillation. Can J Cardiol. 2020;36:1949-55.

8. Shchetynska-Marinova T, Kranert M, Baumann S, et al. Recurrence of atrial fibrillation after pulmonary vein isolation in dependence of arterial stiffness. Neth Heart J. 2021; https://doi.org/10.1007/s12471-021-01644-w.

9. Mol D, Houterman S, Balt JC, et al. Complications in pulmonary vein isolation in the Netherlands Heart Registration differ with sex and ablation technique. Europace. 2021;23:216-25.

10. De With RR, Artola AV, Nguyen BO, et al. Different circulating biomarkers in women and men with paroxysmal atrial fibrillation: results from the AF-RISK and RACE V studies. Europace. 2021; https://doi.org/10.1093/europace/ euab179. 